\title{
VALIDACIÓN DE MÉTODO CUALITATIVO PARA DETECCION DE Staphylococcus aureus Y Streptococcus agalactiae EN MUESTRAS DE LECHE PARA DIAGNÓSTICO DE MASTITIS BOVINA
}

\section{VALIDATION OF QUALITATIVE METHOD FOR THE DETECTION OF Staphylococcus aureus AND Streptococcus agalactiae IN MILK SAMPLES FOR THE DIAGNOSIS OF BOVINE MASTITIS}

\author{
Karen Vargas Hoyos ${ }^{1}$, Juana Vidal Arboleda ${ }^{2}$, Martha Olivera-Angel $^{3}$
}

\begin{abstract}
${ }^{1}$ MyB, Est. M.Sc. Universidad de Antioquia. Facultad de Ciencias Agrarias, Grupo Biogénesis. Carrera 75 No. 65-87, Medellín, Colombia, e-mail: karen.vargas@udea.edu.co; ${ }^{2}$ MyB, M.Sc. Universidad de Antioquia. Facultad de Ciencias Agrarias, Grupo Biogénesis. Carrera 75 No. 65-87, Medellín, Colombia, e-mail: Juana.vidal@udea.edu.co; ${ }^{3}$ DMV, Dr. Sci. Agr. Universidad de Antioquia. Facultad de Ciencias Agrarias, Grupo Biogénesis. Carrera 75 No. 65-87, Medellín, Colombia, e-mail: martha. olivera@udea.edu.co; *Corresponding author
\end{abstract}

Rev. U.D.C.A Act. \& Div. Cient. 21(1): 271-275, Enero-Junio, 2018

https://doi.org/10.31910/rudca.v21.n1.2018.687

\section{INTRODUCCIÓN}

El laboratorio de microbiología veterinaria debe garantizar que los resultados entregados a los usuarios sean válidos y confiables (Herrera et al. 2005) y, para ello, debe realizar un control de calidad, que evalúa y documenta el desempeño de cada uno de los aspectos de sus procesos. Inicia con la calidad de la muestra que recibe, la eficiencia de los reactivos, los medios de cultivo, el funcionamiento de los diversos instrumentos y equipos, el analista y la verificación o validación de los resultados obtenidos. La validación de un método analítico busca determinar, a través de un fundamento estadístico, que sea adecuado, para los fines previstos; se debe realizar de forma ordenada, confiable y trazable, teniendo en cuenta los requerimientos del método y su aplicabilidad, los analitos, las concentraciones y la matriz que se desea utilizar (Pullés et al. 2006; Duffau et al. 2010).

Se conocen dos modalidades o tipos de validación, de acuerdo al método que se emplee: validación primaria, que se define como el proceso exploratorio, que tiene como fin establecer las características de desempeño de un método nuevo o un método clasificado, de forma inadecuada; corresponde a la validación inicial, que deben llevar a cabo los laboratorios o las casas comerciales, que diseñen un método diagnóstico (Camaró et al. 2015; Ruisanchez et al. 2003) y la validación secundaria, se basa en reunir evidencias, que afirmen que el laboratorio está capacitado para cumplir las especificaciones establecidas en la validación primaria; se realiza siempre, cuando se introduce un equipo diagnóstico, método o prueba en un laboratorio, que ha sido validado, primariamente por organizaciones internacionales o por laboratorios de referencia (Camaró et al. 2015).

En el caso del laboratorio microbiológico, los procesos analíticos obligan a tener en cuenta una serie de características propias, que influencian, tanto la forma de validación como el resultado obtenido, por lo tanto, la validación de los métodos microbiológicos debe reflejar las condiciones reales del ensayo y se hace utilizando matrices inoculadas, con un nivel conocido de contaminantes, con el fin de imitar la actividad natural del microorganismo en las muestras (Pérez et al. 2003; Mead et al. 2010). Los tipos de métodos microbiológicos, se pueden clasificar en cualitativos y cuantitativos. Los métodos cualitativos son aquellos en los que se pretende detectar la existencia o ausencia de un microorganismo determinado en una muestra. El método cuantitativo es en el que se desea indicar el número de unidades formadoras de colonias en una cantidad de muestra, realizando recuentos y cuantificaciones y su objetivo es detectar el valor numérico de un agente infeccioso en una muestra (Linossier et al. 2003). En los métodos cualitativos, los objetivos de la validación son asegurar que se detecten las muestras positivas hasta un nivel de positividad bajo y que no se presenten falsos positivos, los que podrían dar lugar a un resultado erróneo (Camaró et al. 2015). 
Los parámetros de un método cualitativo son las características del mismo, que lo hacen apto para un uso previsto: a) límite de detección o mínima cantidad de microorganismo que puede ser determinada por el método: es la manera de asegurar que este es capaz de detectar e identificar la presencia de microorganismos diana, en muestras con poca carga microbiana y b) relativos a una correcta detección del método: sensibilidad, especificidad, falsos positivos, falsos negativos (Suarez et al. 2009; Sánchez et al. 2010).

El objetivo de este estudio fue realizar la validación secundaria del método cualitativo utilizado para la detección de Staphylococcus aureus y Streptococcus agalactiae, en muestras de leche bovina, sospechosas de mastitis.

\section{MATERIALES Y MÉTODOS}

Evaluación cepas: Se evaluaron un total de seis cepas a saber: Cepa Patrón: Cepa de S. aureus ATCC 29213, Cepa de S. agalactiae ATCC 12383; Cepa Diana: Cepas del microorganismo $S$. aureus y de $S$. agalactiae aislado en el laboratorio; Cepa no diana: Cepas diferentes a S. aureus y $S$. agalactiae aislados en el laboratorio.

Selección de la matriz: Se usó leche ultra alta temperatura (UAT), por sus características de inocuidad y de calidad, que permiten asegurar que el microorganismo inoculado será el único recuperado en los ensayos de validación.

Preparación del inóculo: Los cultivos utilizados, se encontraron en fase estacionaria, los que se obtienen sembrando la cepa deseada (diana) y no deseada (no diana) por agotamiento (facilita la separación y el aislamiento de las colonias), en agar sangre, durante 24 a 48 horas, a $37^{\circ} \mathrm{C} \pm 2$, en ambiente anaerobio facultativo, con 5\% $\mathrm{CO}_{2}$. Una vez obtenido el cultivo de ambas cepas, se hicieron 10 réplicas de 10 diluciones, desde $10^{-1}$ hasta $10^{-10}$ en agua peptonada, al $0,1 \%$. Se sembró $1 \mathrm{~mL}$ de cada dilución en agar sangre y se incubó durante 24 horas, a $37^{\circ} \mathrm{C} \pm 2,5 \%$ de $\mathrm{CO}_{2}$. Finalizado este tiempo, se contó el número de unidades formadoras de colonia (UFC) en cada dilución y se tomó la que contenía 5 a 10 UFC, con las que se inoculó la matriz.

Parámetros a medir: Límite de detección: La matriz (leche (UAT), se inoculó con las cepas patrón y se hicieron diluciones desde $10^{-1}$ hasta $10^{-10}$, partiendo de la concentración McFarland ( $9 \mathrm{ml}$ de leche UHT $+1 \mathrm{~mL}$ del inóculo, se repite con cada dilución). Se sembró cada dilución en agar sangre y se incubó a $37^{\circ} \mathrm{C} \pm 2$, en ambiente de $5 \% \mathrm{CO}_{2}$, durante 24 horas y se interpretaron los datos; en cada dilución sembrada, se anotó el número de UFC y se determinó cuál es la dilución mínima, en la que hubo crecimiento. El límite de detección fue la concentración inmediatamente anterior, a la que no se obtuvo ningún crecimiento, según la norma ISO 19036:2006 para alimentos de consumo animal. Pruebas Tamiz: Para esta, se sembró 3 veces cada cepa en agar sangre, para un total de 18 siembras (Cepa ATCC, cepa diana y cepa no diana), se incubó $24 \mathrm{~h}$, se registró si hubo o no crecimiento; a cada una de las cepas en las que se obtuvo crecimiento, se realizó: coloración de Gram, según instructivo "Coloración de Gram", pruebas de identificación para Staphylococcus y pruebas de identificación para Streptococcus".

Método de referencia: El BD BBL Crystal ${ }^{\mathrm{TM}}$ Identificación Systems Gram-Positive ID kit, límite establecido con el Factor McFarland 0,5.

Tabla de contingencia: Se usó tabla de contingencia de 2x2, para la realización de los análisis.

Cálculo de la sensibilidad: A partir de la prueba tamiz, se calculó la capacidad del método, para mostrar verdaderos positivos, cuando la muestra contenía el analito: \% Sensibilidad $=(\mathrm{VP} / \mathrm{VP}+\mathrm{FN}) * 100$. Criterio de aceptación: el resultado fue válido, cuando el porcentaje de sensibilidad fue mayor al 95\%.

Cálculo de la Especificidad: A partir de la prueba tamiz, se calculó la capacidad del método de mostrar verdaderos negativos, cuando las muestras no tenía el analito: \% Especificidad $=(\mathrm{VN} / \mathrm{VN}+\mathrm{FP}) * 100$. Criterio de aceptación: el resultado fue válido, cuando el porcentaje de especificidad es mayor al $95 \%$.

Determinación del valor predictivo positivos (VPP): Se calculó probabilidad de que una muestra con valor verdaderamente positivo (VPP) (método de referencia) dé un valor positivo, por el método a validar: $\mathrm{VPP}=\mathrm{VP} /(\mathrm{VP}+\mathrm{FP})$. El criterio de aceptación: si $\mathrm{FP}<0,95$ el método analítico no tiene buen valor predictivo positivo. Si FP $\geq 0,95$, el método analítico tiene buen VPP.

Determinación del valor predictivo negativos (VPN): Se analizó la probabilidad de que una muestra con valor verdaderamente negativo (método de referencia) de un valor negativo, por el método a validar: $\mathrm{VPN}=\mathrm{VN} /(\mathrm{VN}+\mathrm{FN})$. $\mathrm{El}$ criterio de aceptación: si FN $<0.95$ el método analítico no tiene buen VPN. Si FN $\geq 0.95$, el método analítico tiene buen VPN.

\section{RESULTADOS Y DISCUSIÓN}

Como resultado de los parámetros medidos del método cualitativo en $S$. agalactiae y en $S$. aureus, se encontró que el límite de detección para $S$. agalactiae es de $10^{-7}$ UFC y para $S$. aureus, de $10^{-9}$ UFC (Imagen 1). A partir de los resultados obtenidos, se evidencia que el límite de detección para $S$. aureus es mayor que para $S$. agalactiae, concordando con 

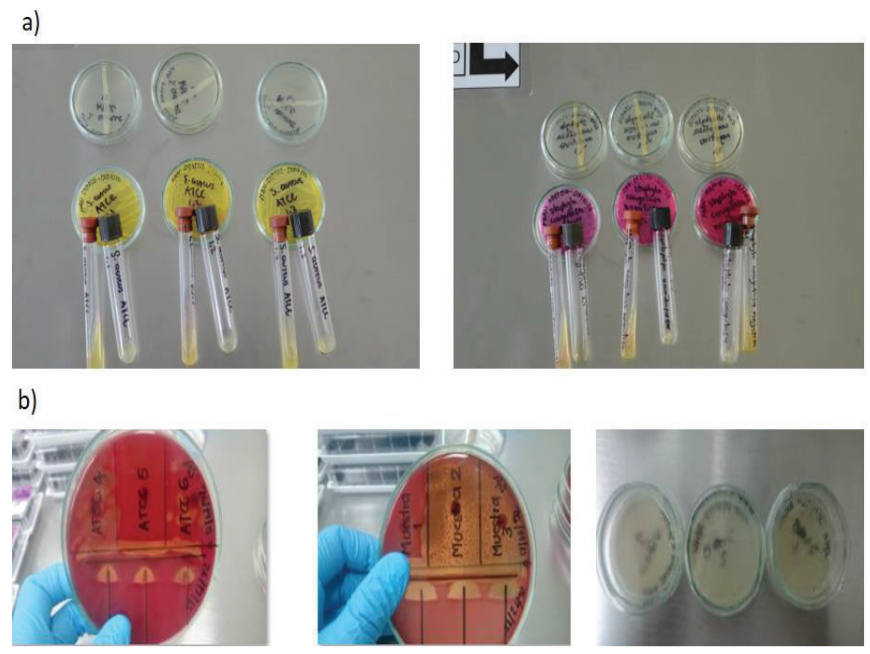

Imagen 1. a) Pruebas realizadas para la identificación de Staphylococcus aureus (coagulasa positivo) y b) Pruebas realizadas para la identificación de Streptococcus agalactiae (CAMP: Positivo).

Tabla 1: Tabla de contingencia y de resultados para Streptococcus agalactiae y para Staphylococcus aureus.

\begin{tabular}{|c|c|c|c|c|}
\hline \multirow{2}{*}{\multicolumn{2}{|c|}{ a) Resultados del ensayo }} & \multicolumn{2}{|c|}{ Recuento confirmado } & \multirow{3}{*}{$\begin{array}{c}\text { Total } \\
6\end{array}$} \\
\hline & & \multirow{2}{*}{$\frac{+}{6(\text { S. agalactiae })}$} & \multirow{2}{*}{$\begin{array}{l}- \\
0\end{array}$} & \\
\hline Recuento & + & & & \\
\hline Presuntivo & - & 0 & 12 & 12 \\
\hline \multicolumn{2}{|c|}{ Total } & 6 & 12 & \\
\hline \multirow{2}{*}{\multicolumn{2}{|c|}{ b) Resultados del ensayo }} & \multicolumn{2}{|c|}{ Recuento confirmado } & \multirow{2}{*}{ Total } \\
\hline & & + & - & \\
\hline \multirow{2}{*}{$\begin{array}{l}\text { Recuento } \\
\text { Presuntivo }\end{array}$} & + & 6 (S. aureus) & 0 & 6 \\
\hline & - & 0 & 12 & 12 \\
\hline \multicolumn{2}{|c|}{ Total } & 6 & 12 & \\
\hline \multicolumn{5}{|l|}{ c) } \\
\hline \multicolumn{2}{|c|}{$\begin{array}{l}\% \text { Sensibilidad }= \\
(V P / N P+F N) * 100\end{array}$} & \multicolumn{3}{|l|}{$(6 / 6) * 100=100 \%$} \\
\hline \multicolumn{2}{|c|}{$\begin{array}{l}\% \text { Especificidad }= \\
(\text { VN/VN+FP) } * 100\end{array}$} & \multicolumn{3}{|c|}{$(12 / 12) * 100=100 \%$} \\
\hline \multicolumn{2}{|c|}{$\mathrm{VPP}=\mathrm{VP} /(\mathrm{VP}+\mathrm{FP})$} & \multicolumn{3}{|l|}{$6 /(6+0)=1$} \\
\hline \multicolumn{2}{|c|}{$\mathrm{VPN}=\mathrm{VN} /(\mathrm{VN}+\mathrm{FN})$} & \multicolumn{3}{|l|}{$12 /(12+0)=1$} \\
\hline
\end{tabular}

a) Resultados obtenidos para Streptococcus agalactiae. b) Resultados obtenidos para Staphylococcus aureus. c) Resultados para ambos microorganismos. Todas las cepas se probaron en el método de referencia BBL Crystal para Gram positivos. 
los resultados obtenidos por Riekerink et al. (2006), quienes aislaron $S$. aureus, en un $74 \%$, mientas que $S$. agalactiae, en un $1,6 \%$, lo que sugiere que $S$. aureus posee propiedades microbiológicas, que favorecen su tasa de crecimiento, de ahí que la recuperación de $S$. aureus sea mayor que la de S. agalactiae (Riekerink et al. 2006; Elhaig \& Selim, 2015).

La sensibilidad y la especificidad determinada por medio de la tabla de contingencia, demostró que el método evaluado logró determinar el género y la especie de las 6 cepas, tanto de S. aureus (cepa ATTCC y cepas diana) como de $S$. agalactiae (cepa ATTCC y cepas diana), con una sensibilidad y especificidad del $95 \%$, indicando que el método utilizado detecta las cepas verdaderamente positivas y excluye las verdaderamente negativas (Imagen 1) (Tabla 1). La evaluación de estos parámetros garantiza que la identificación del microorganismo es confiable y veraz, similar a lo encontrado por Buelow et al. (1996), quienes demuestran que el cultivo de leche puede ser altamente sensible, con un rango 91$100 \%$ (Tabla 1). Igualmente, los resultados de sensibilidad y de especificidad encontrados en este estudio con relación S. agalactiae son similares a los reportados por Keefe et al. (1997) y Sawant et al. (2002), quienes obtuvieron altos porcentajes (91\%) de sensibilidad y de especificidad, aun cuando se utilizan métodos de identificación diferentes al evaluado en este trabajo.

Al realizar la comparación de los resultados con el método elegido de referencia BD BBL Crystal ${ }^{\mathrm{T}}$ Identification Systems Gram-Positive ID kit, las cepas coinciden en la identificación obtenida por los dos métodos (Altman \& Bland, 1994; Camaró et al. 2015) (Tabla 1). El método referencia BD BBL Crystal tuvo un desempeño acorde con las especificaciones dadas en la guía de procedimiento del mismo, garantizando, con ello, la confiabilidad en los resultados, del 99\% (OIE, 2009). A través de los valores predictivos, se obtiene un presuntivo de resultado para cada prueba; estudios teóricos han sugerido que la suma de la sensibilidad y de la especificidad debe tener un valor de, al menos, $100 \%$ de precisión, para dar la seguridad de ser un buen predictor (Linossier et al. 2003); en este caso, la suma de estos dos valores fue de $90 \%$, indicando un alto valor predictivo. Este método y los parámetros analizados garantizan, en gran medida, la detección correcta del microorganismo, en el laboratorio de microbiología veterinaria (Altman \& Bland, 1994; Sánchez et al. 2010).

En función de los resultados obtenidos, se puede concluir que, el método propuesto de aislamiento e identificación de Staphylococcus aureus y Streptococcus agalactiae, a partir de muestras de leche, es capaz de recuperar concentraciones bajas de los microorganismos y, dado que presenta valores de sensibilidad y de especificidad del $90 \%$, hacen que este método resulte confiable y, a la vez, que garantiza una correcta identificación de S. aureus y de S. agalactiae, patógenos importantes en la mastitis bovina.

Agradecimientos: A la Universidad de Antioquia, Facultad de Ciencias Agrarias, Unidad de Diagnóstico, grupo de investigación Biogénesis. Conflictos de intereses: El manuscrito fue preparado y revisado con la participación de todos los autores, quienes declaramos que no existe ningún conflicto de intereses que ponga en riesgo la validez de los resultados presentados. Financiación: Este estudio fue financiado por grupo de investigación Biogénesis, Universidad de Antioquia.

\section{BIBLIOGRAFÍA}

1. ALTMAN, D.G.; BLAND, J.M. 1994. Diagnostic Tests 1: Sensitivity and Specificity. British Medical Journal BMJ (United Kingdom). 308(6943):1552-1553.

2. ALTMAN, D.G.; BLAND, J.M. 1994. Diagnostic tests 2: predictive values. British Medical Journal BMJ (United Kingdom). 309(6947):102.

3. BUELOW, K.L.; THOMAS, C.B.; GOODGER, W.J.; NORDLUND, K.V.; COLLINS, M.T. 1996. Effect of milk sample collection strategy on the sensitivity and specificity of bacteriologic culture and somatic cell count for detection of Staphylococcus aureus intramammary infection in dairy cattle. Preventive Veterinary Medicine (United States). 26(1):1-8.

4. CAMARÓ, M.L.; MARTÍNEZ, R.; OLMOS, P.; CATALÁ, V.; OCETE, M.D.; CARDONA, C. 2015. Validación y verificación analítica de los métodos microbiológicos. Enfermedades Infecciosas y Microbiología Clínica (España). 33(7):e31-e36.

5. DUFFAU, B.; ROJAS, F.; GUERRERO, I.; ROA, L.; RODRÍGUEZ, L.; SOTO, M.; SANDOVAL, S. 2010 Validación de métodos y determinación de la incertidumbre de la medición: "aspectos generales sobre la validación de métodos". Guía técnica (Chile). (1):2149.

6. HERRERA, M.L.; CAMPOS, M. 2005. Control de la Calidad para un Laboratorio de Microbiología Introducción Elementos mínimos del Programa de Control de Calidad en Microbiología. Rev. Médica Hospital Nacional de Niños Dr. Carlos Sáenz Herrera (Costa Rica). 40(1):9-15.

7. KEEFE, G.P.; DOHOO, I.R.; SPANGLER, E. 1997. Herd prevalence and incidence of Streptococcus agalactiae in the dairy industry of Prince Edward Island. Journal of Dairy Science (Canada). 80(3):464-470. 
8. LINOSSIER, A.; VARGAS, A.; ZILLMANN, G.; ARRIAGADA, M.; ROJAS, R.; VILLEGAS, R. 2003. Streptococci mutans: Método semi-cuantitativo para establecer el rango de riesgo de infección bucal en niños preescolares chilenos. Rev. Médica de Chile (Chile). 131(4):412-418.

9. ELHAIG, M.M.; SELIM, A. 2015. Molecular and bacteriological investigation of subclinical mastitis caused by Staphylococcus aureus and Streptococcus agalactiae in domestic bovids from Ismailia, Egypt. Tropical animal health and production (United Kingdom). 47(2):271-276.

10. MEAD, G.; LAMMERDING, A.M.; COX, N.; DOYLE, M.P.; HUMBERT, F.; KULIKOVSKIY, A.; SALMONELLA ON RAW POULTRY WRITING COMMITTEE. 2010. Scientific and technical factors affecting the setting of Salmonella criteria for raw poultry: a global perspective. J. Food Protection (United States). 73(8):1566-1590.

11. OIE. 2009. Principios y métodos de validación de las pruebas de diagnóstico de las enfermedades infecciosas. En: Manual Acuático de la OIE 2012. Disponible desde Internet en: http://www.oie.int/esp/normes/fmanual/1.1.02_VALIDATION.pdf (con acceso 08/02/2018).

12. PÉREZ, D.; CAMPOS, L.; DOMÍNGUEZ, I.; SOSA, A.E. 2003. Verificación rápida de la pureza microbiológica de bancos de Escherichia coli K12. Biotecnologia aplicada (Cuba). 20(4):231-237.

13. PULLÉS, M.R.; NAVARRO, R.M.; MORALES, V.M. 2006. Criterios para la acreditación de laboratorios que realizan ensayos microbiológicos según NC-ISO/ IEC 17025: 00. Revista CENIC Ciencias Biológicas (Cuba). 37(1):32-36.

14. RIEKERINK, R.G.O.; BARKEMA, H.W.; VEENSTRA, S.; POOLE, D.E.; DINGWELL, R.T.; KEEFE, G.P. 2006. Prevalence of contagious mastitis pathogens in bulk tank milk in Prince Edward Island. The Canadian Veterinary J. 47(6):567.

15. RUISANCHEZ, I.; TRULLOLS, E.; RIUS, F.X. 2003. Validación de métodos analíticos cualitativos. Técnicas de laboratorio(España). (81):328-335.

16. SÁNCHEZ, J.F.; TEJEDA, M.E.; KOCH, W.; MORA, J.L.A.; MARROQUÍN, R.; HERNÁNDEZ, V.; ISLAS, V.; SÁNCHEZ, E.G.; LEÓN, A.D. 2010. Validación de métodos analíticos no cuantitativos. Revista Mexicana de Ciencias Farmacéuticas (México). 41(2):15-24.

17. SAWANT, A.A.; PILLAI, S.R.; JAYARAO, B.M. 2002. Evaluation of five selective media for isolation of catalase-negative gram-positive cocci from bulk tank milk. Journal of dairy science (Canada). 85(5):1127-1132.

18. SUAREZ, R.; ARÉVALO, E.; LINARES, L.; USTÁRIZ, F.; HERNÁNDEZ, G. 2009. Validación de un método analítico para la determinación de magnesio eritrocitario. Avances en Química (Venezuela). 4(2):53-62.

Recibido: Enero 9 de 2018

Aceptado: Mayo 16 de2018

Cómo citar:

Vargas Hoyos, K.; Vidal Arboleda, J.; Olivera-Ángel, M. 2018. Validación de método cualitativo para detección de Staphylococcus aureus Y Streptococcus agalactiae en muestras de leche para diagnóstico de mastitis bovina. Rev. U.D.C.A Act. \& Div. Cient. 21(1): 271-275. 